\title{
Effects of instrument settings on radiofrequency ultrasound local estimator images: A preliminary study in a gallbladder model
}

\author{
JIAN WANG, CHUNSONG KANG, TINGHUA FENG, JIPING XUE, KAILING SHI, \\ TINGTING LI, XIAOFANG LIU and YU WANG
}

Department of Ultrasound, The First Hospital of Shanxi Medical University, Taiyuan, Shanxi 030001, P.R. China

Received December 12, 2012; Accepted April 18, 2013

DOI: $10.3892 / \mathrm{mmr} .2013 .1613$

\begin{abstract}
The aim of the present study was to evaluate the changes in radiofrequency ultrasound local estimator (RULES) images with different instrument settings. An Esaote Technos MPX Color Doppler Ultrasound Machine and RULES were used to capture images of a gallbladder model. The percentages of various colored areas (color filling rates) within the area of interest were calculated using different instrument gains, transducer frequencies and scan depths. Blue was predominant in the lumen of the model gallbladder, while red and green were primarily located near the inner edge of the lumen. When the depth was set at $62 \mathrm{~mm}$ and the gain at 105 , the total color filling rates did not vary with different transducer frequencies. The blue color filling rate was greatest with a transducer frequency of $8.0 \mathrm{MHz}$, and the red and green color filling rates were greatest with a frequency of $12.5 \mathrm{MHz}$. Color variety was greatest when the transducer frequency was 12.5 MHz. When the transducer frequency was $12.5 \mathrm{MHz}$ and the depth was $62 \mathrm{~mm}$, the blue color filling rate was greatest with gains of 105 and 110, the red color filling rate was greatest with gains of 95 and 100 and the green color filling rate was greatest when the gain was 100 . The total color filling rate was greatest at gains of 100 and 105. In conclusion, images obtained using RULES may be affected by the instrument gain and, to a certain extent, by transducer frequency.
\end{abstract}

\section{Introduction}

Ultrasonic tissue characterization (UTC) is a method for exploring the association between the acoustic properties and ultrasonic manifestations of tissues (1). UTC utilizes tissue status-reflecting parameters that may be isolated from ultrasound signals in order to distinguish different tissues and to identify the nature of lesions (2). Conventional two-dimen-

Correspondence to: $\mathrm{Dr}$ Chunsong Kang, Department of Ultrasound, The First Hospital of Shanxi Medical University, 85 Jie Fang Nan Road, Taiyuan, Shanxi 030001, P.R. China

E-mail: chunsongkang57@126.com

Key words: ultrasound, radio frequency, tissue characterization, frequency, radiofrequency ultrasound local estimator sional (2D) ultrasound imaging reflects the anatomical structure of cross-sections in grayscale, and the shade depends on the echo amplitude. However, the echo amplitude is the external envelope of the original signals, and tissue imaging based on signal envelope extraction is unable to reveal the detailed characteristics of tissues (3).

Radiofrequency ultrasound local estimators (RULES) is a newly developed tissue characterization method (4-7). When ultrasonic energy passes through soft tissue, its mechanical energy interacts with local tissues, and energy absorption, wave reflection and dispersion occur simultaneously. The energy that is reflected back to the ultrasonic transducer constitutes the ultrasonic echo signals (8). The ultrasonic signals received on the surface of the transducer are formed by the overlapping of waves with different amplitudes, and their duration and overlap are related to their ultrasound bandwidth and center frequency. Signals received in this form are the original signals $(3,7)$.

Original signals contain a large amount of information with regard to the tissue characteristics, and the RULES algorithm is based on interactive radiofrequency (RF) signal processing. RULES echographic signals are processed by fast echographic multiparameter multi-image novel apparatus (FEMMINA), a newly developed hardware/software platform that extracts the characteristic parameters of local tissues using the wavelet analysis method for texture characterization, and shares the image processing between the hardware and software. The original signals are converted into different colors that represent different tissue structural information after being analyzed and processed by RULES.

The RULES method, although still in its infancy, has been shown to have a potential clinical application for the diagnosis of prostate $(9,10)$ and breast cancer $(8)$, and the differentiation of carotid plaque tissue (11). However, similar to conventional ultrasonographic tissue characterization, images obtained using the RULES method may be affected by variables, including transducer frequency, instrument gain and scanning depth. Limited research has been carried out on the potential effects of differences in these imaging parameters on RULES images. Thus, the aim of the present study was to investigate the effects of probe frequency, gain and scanning depth on the characteristics of RULES images, using a phantom gallbladder model. This model was selected in order to eliminate the effects of clinical variables on the images. 


\section{Materials and methods}

Model. The experiment was performed using the gallbladder model in the Abdominal Intraoperative and Laparoscopic Ultrasound Phantom (Kyoto Kagaku Co., Kyoto, Japan; http:// www.kyotokagaku.com/products/detail03/us-3.html). The study was approved by the Ethics Committee of The First Hospital of Shanxi Medical University, Taiyuan, P.R. China.

Instruments and methods. An Esaote Technos MPX Color Doppler Ultrasound Machine (Esaote, Genova, Italy) was used; this was connected to a rapid analysis multi-image multi-parameter parallel processor through an optical fiber. The transducer used was an LA523 broadband transducer (Esaote) with a frequency of 4-13 MHz. One experienced ultrasonographer performed all of the examinations in this study.

Image acquisition. Firstly, conventional 2D ultrasound was used to scan the simulated gallbladder in the model, and the position and size of the simulated gallbladder were observed. The device was then switched to the RULES functional status, and the mechanical index (MI) was fixed at 0.7. The time gain compensation (TGC) was kept constant at the near and far fields, and the focus was located at the center of the image. The settings were saved to ensure consistent test conditions.

The total gain was fixed at 110, the depth was fixed at $62 \mathrm{~mm}$ and images output by the FEMMINA platform were obtained with transducer frequencies of 5.5, 8.0, 10.0 and $12.5 \mathrm{MHz}$. The transducer frequency was then set at $12.5 \mathrm{MHz}$ and the depth was fixed at $62 \mathrm{~mm}$; images were captured with the total gain set at 90, 95, 100, 105, 110, 115, 120, 125 and 130. Thereafter, the total gain was fixed at 110 , the transducer frequency was set at $12.5 \mathrm{MHz}$ and images were captured for depths of 62, 72, 83 and $103 \mathrm{~mm}$. In all instances, the position of the transducer remained unchanged during the image acquisition process. A total of 20 images were obtained for each set of parameters. In order to ensure objectivity, in each case, 4 images were captured and the system was exited. This image acquisition process was repeated 5 times to obtain a total of 20 images for each unique combination of machine settings.

A frequency of $12.5 \mathrm{MHz}$ was selected, since the images exhibited the greatest number of colors at this frequency. A depth of $62 \mathrm{~mm}$ was selected based on the penetration force of the ultrasound and the display range; a depth of $<62 \mathrm{~mm}$ decreased the display range to a degree that the complete gallbladder was not included. A depth of $>62 \mathrm{~mm}$ resulted in insufficient penetration of the ultrasound, and the gallbladder was not clearly displayed. The gain was set at 110 as the filling rate of the images was efficient at this gain, as determined by visual estimation, and it was the median of the range of gains studied.

Image measurement and analysis. The Image-Pro Plus analysis software (Media Cybernetics, Bethesda, MD, USA) was used to analyze the captured images. The area of interest (AOI) was drawn along the inner edge of the simulated gallbladder, and the software automatically calculated the percentages of various colored areas in the AOI. After selecting the color to be analyzed, the software automatically calculated the percentage of that color in the AOI of the simulated gallbladder, which was defined as the color filling rate.
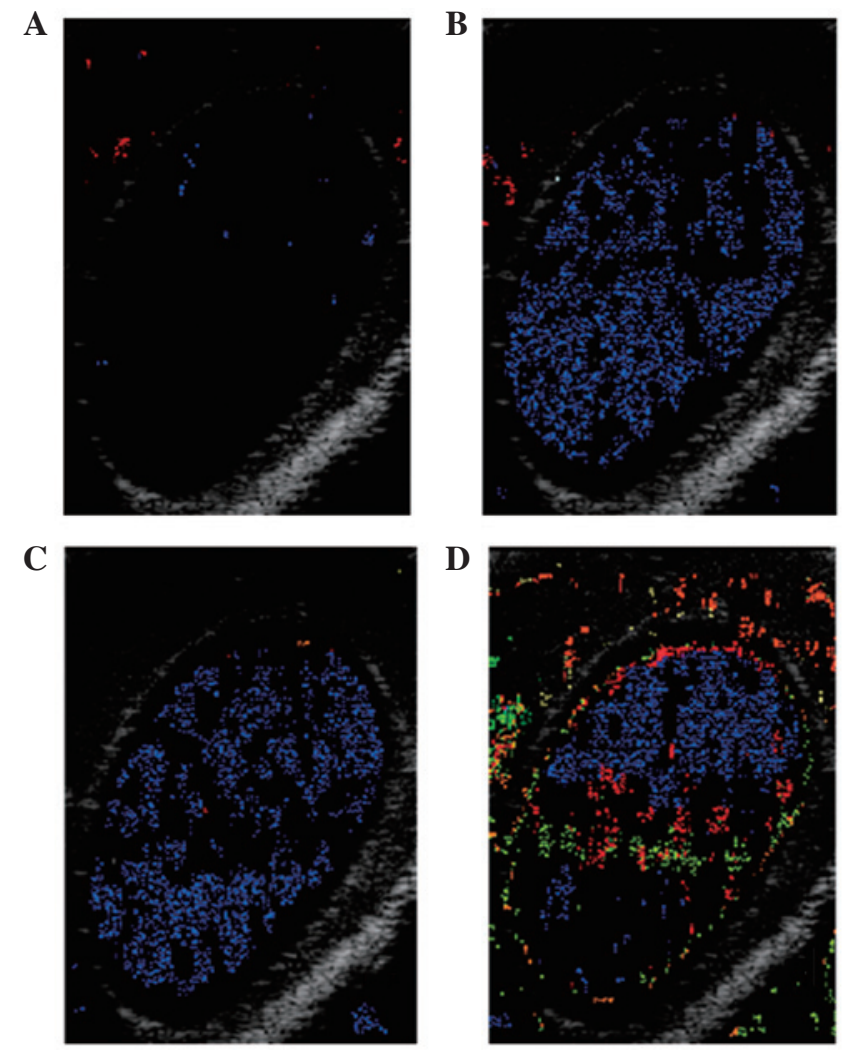

Figure 1. Color filling map of the simulated gallbladder at different frequencies when the depth was $62 \mathrm{~mm}$ and the gain was 110. (a) Transducer frequency, 5.5 MHz; almost no color filling was present. (b) Transducer frequency, $8.0 \mathrm{MHz}$; the blue color filling rate was high and the color was mainly blue. (c) Transducer frequency, $10.0 \mathrm{MHz}$; the blue color filling was predominant. (d) Transducer frequency, $12.5 \mathrm{MHz}$; multiple colors were observed.

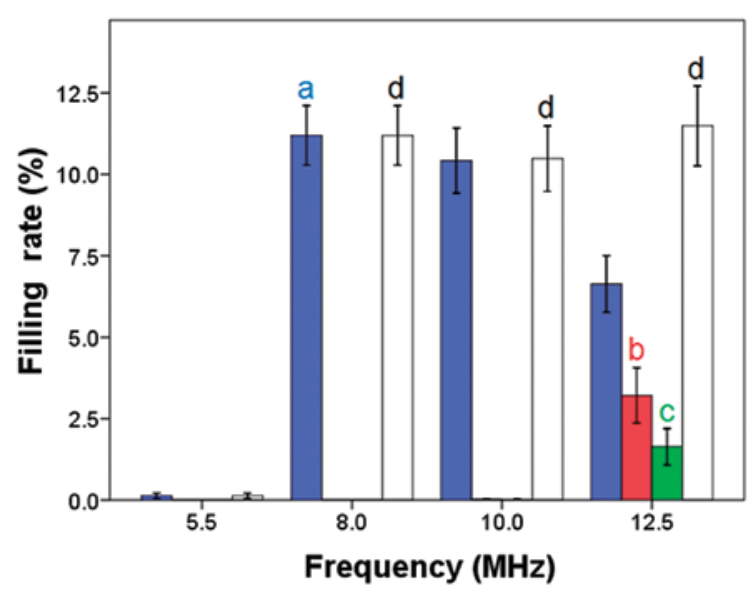

Figure 2. Effects of transducer frequency on the color filling rate. Data are presented as the mean \pm standard deviation (SD) for each color. Blank bars represent the total color filling rates. ${ }^{\mathrm{a}-\mathrm{d}} \mathrm{P}<0.05$ (significant increase) compared with the other frequency levels of the same color, as determined using Tukey's adjustment.

Statistical analysis. Results for color filling rate are presented as the mean \pm standard deviation (SD). Comparisons between groups were evaluated using ANOVA with Tukey's adjustment. Data analyses were performed using the SAS version 9.0 statistical software (SAS Institute Inc., Cary, NC, USA). P $<0.05$ was considered to indicate a statistically significant difference. 


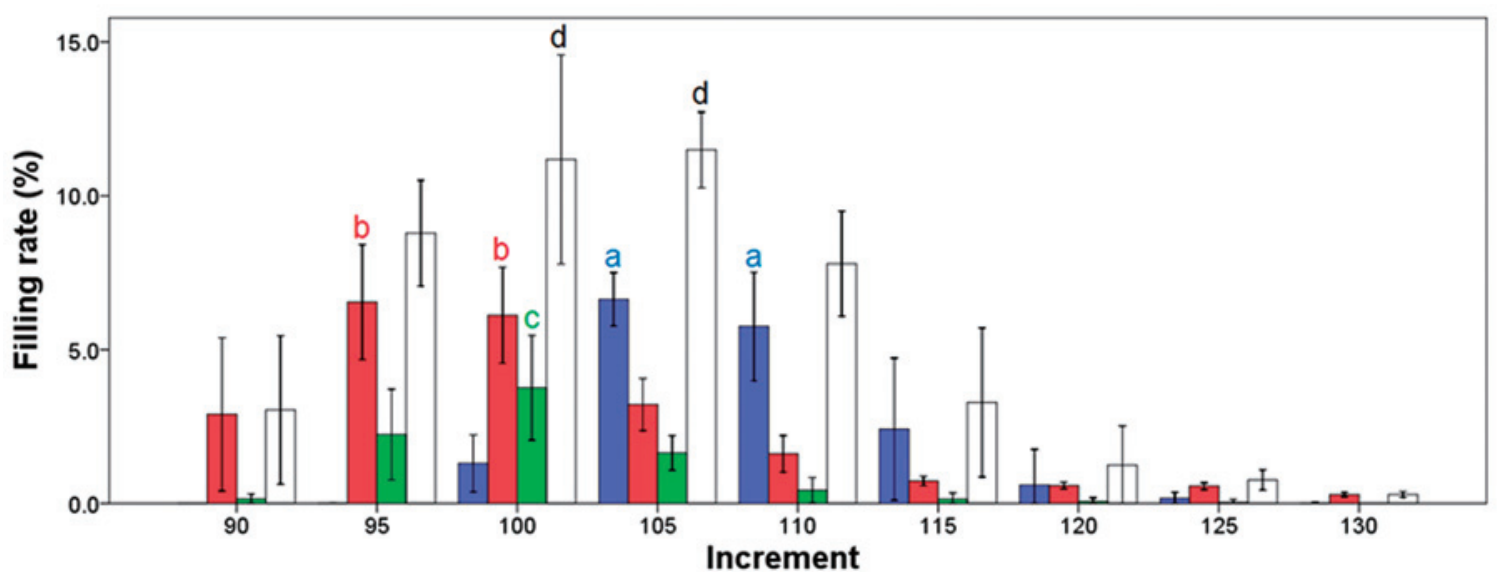

Figure 3. Effects of gain on the color filling rate when the depth was $62 \mathrm{~mm}$ and the frequency was $12.5 \mathrm{MHz}$. The blue color filling rate was highest at gains of 105 and 110, the red color filling rate was highest at gains of 95 and 100, and the green color filling rate was highest at a gain of 100. The total color filling rate was highest within the range of 95-110. Data are presented as the mean \pm standard deviation (SD) for each color. Blank bars represent the total color filling rates. ${ }^{\mathrm{a}-\mathrm{d}} \mathrm{P}<0.05$ (significant increase) compared with the other increment levels of the same color, as determined using Tukey's adjustment.

\section{Results}

Images. Images of the simulated gallbladder output by FEMMINA manifested as a superposition of conventional 2D images and color images after being processed using the RULES method. The colors were blue, red (or red-orange) and green (or green-yellow). Blue was predominant in the lumen of the model gallbladder, while red and green were primarily located near the inner edge of the lumen (Fig. 1).

Effects of transducer frequency on the color filling rate. When the depth was set at $62 \mathrm{~mm}$ and the gain at 105, the total color filling rate in the simulated gallbladder was similar at transducer frequencies of $8.0,10.0$ and $12.5 \mathrm{MHz}$; no statistically significant differences were observed $(\mathrm{P}=0.0936$, $\mathrm{P}=1.00$ and $\mathrm{P}=0.069$, compared with $5.5 \mathrm{MHz}$, respectively; Figs. 1 and 2). The blue color filling rate was greatest with a transducer frequency of $8.0 \mathrm{MHz}$, while the red and green color filling rates were greatest with a frequency of $12.5 \mathrm{MHz}$. Additionally, the color variety was greatest when the transducer frequency was $12.5 \mathrm{MHz}$.

Effects of gain on the color filling rate. Color filling rate data with a fixed depth and transducer frequency are shown in Fig. 3. When the transducer frequency was $12.5 \mathrm{MHz}$ and the depth was $62 \mathrm{~mm}$, the blue color filling rate was greatest at gains of 105 and 110; no significant differences were identified between the values $(\mathrm{P}=0.51)$. When the gain was $<105$ or $>110$, the blue color filling rate was decreased (all $\mathrm{P}<0.0001$ ). The red color filling rate was greatest at gains of 95 and 100; no significant difference was identified $(\mathrm{P}=1.00)$. When the gain was $<95$ or $>100$, the red color filling rate was decreased (all $\mathrm{P}<0.0001)$. The green color filling rate was greatest when the gain was 100 , and was significantly greater than that at gains of 95 and 105 (both $\mathrm{P}<0.0001$ ). When the gain was $<95$ or $>105$, the green color filling rate was decreased (all $\mathrm{P}<0.0001$ ). The total color filling rate was greatest at gains of 100 and 105, and was significantly greater compared with that at gains of 95 and 110 (both $\mathrm{P}<0.0001$ ). When the gain was $<95$ or $>110$, the total color filling rate was decreased (all $\mathrm{P}<0.0001$ ).

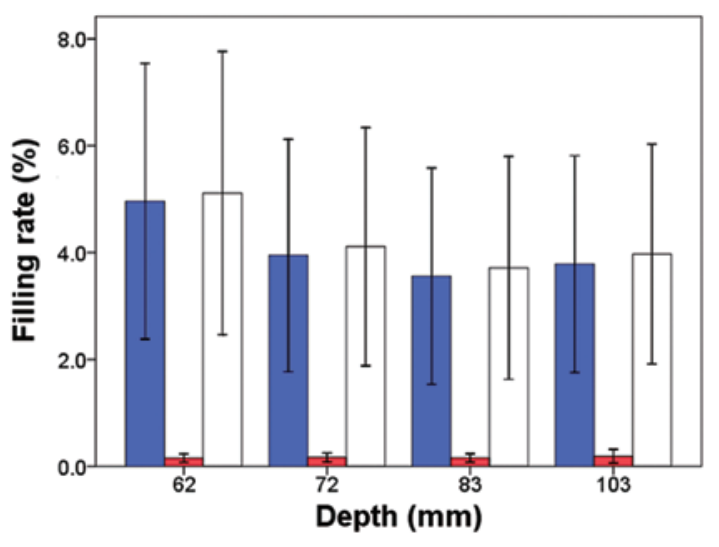

Figure 4. Effects of depth on the color filling rate when the frequency was $10.0 \mathrm{MHz}$ and the gain was 110 . The blue color filling was predominant, and there was no difference among the groups $(P>0.05)$. Data are presented as the mean \pm standard deviation (SD) for each color. Blank bars represent the total color filling rates.

Effects of scan depth on the color filling rate. When the gain was 110 and the transducer frequency was $10 \mathrm{MHz}$, the color filling rates of scan depths ranging from $62-103 \mathrm{~mm}$ were not significantly different (Fig. 4).

\section{Discussion}

The basic process of the UTC method may be described by the following steps: i) Radiofrequency signal acquisition and local spectrum processing; ii) statistical analysis of local characterization parameters and construction of the corresponding database; iii) comparison of the local characterization parameters and the database, and determination of the pathological characteristics of the tissues; and iv) display of the characteristic as a superposition of 2D and color images (8).

RULES produces a final image in which different structural organizations of the tissue being scanned are represented by different colors superimposed on a conventional B-mode image. RULES efficiently reflects the structural characteristics of tissues (7); therefore, it is considered to have a potential for broad clinical applications. However, although the actual images 
obtained through RULES are associated with tissue structures, they may be affected by instrument factors, including transducer frequency, scan depth and total gain. To the best of our knowledge, no previous studies on the RULES method have provided data regarding the potential effects of these parameters on the images obtained. In the present study, we found that images obtained using RULES may be affected by instrument gain, and, to a certain extent, by transducer frequency.

Although in its infancy, preliminary studies have demonstrated the potential clinical application of RULES and the FEMMINA platform. Bertaccini et al (10) performed a double-blind prospective study using 105 patients with clinical or biochemical findings indicating the presence of prostate cancer. All the patients underwent transrectal biopsy with ultrasonography using the FEMMINA platform that processes signals by a RULES algorithm. Of the 32 patients with histologically diagnosed cancer, 26 were found to be positive using the RULES method. RULES exhibited an improved positive and negative predictive value, sensitivity, specificity and accuracy compared with B-mode ultrasound. In an in vivo experiment by Masotti et al (8), it was reported that RULES was able to accurately distinguish breast carcinoma, fibroademona and cysts. Masotti et al (11) also investigated the use of RULES for the differentiation of carotid plaque tissues, and found that it was possible to accurately distinguish between calcifications, blood and lipids, and necrosis.

The implementation of RULES relies on support from the FEMMINA hardware and software platform (11). The software used in this study was based on the tissue characterization database and the captured images reflected the RF signals of local tissues, which were affected by the transducer frequency and, to a certain extent, by the total gain.

The results of the present study showed that the color filling rates of RULES images are affected by the total gain and, to a certain extent, by the transducer frequency. The greatest numbers of colors were displayed at a frequency of $12.5 \mathrm{MHz}$, which is likely to be due to the higher frequency (i.e., a higher frequency contains an increased amount of information and thus better resolution). No obvious color filling of the images was observed at $5.5 \mathrm{MHz}$, which may be associated with insufficient resolution, even though the penetrating force was adequate. When the frequency was 8.0 and $10.0 \mathrm{MHz}$, the blue color filling rate of the images was high, the color variety was limited and the color filling rates of other colors were low. There were no significant differences in the total color filling rates at 8.0,10.0 and $12.5 \mathrm{MHz}$.

In the present study, RULES images were also found to be affected by the total instrument gain. Gain represents the amplification of the signals received by the transducer; the greater the gain, the greater the brightness of the images. However, greater gain results in increased signal noise. When the total gain was between 95 and 110, the color filling rate of the images was relatively higher and constant. When the gain was excessively high, the color filling rates of all the colors were low. This finding is likely to be associated with the increase of noise with higher gains. When the gain was low, a decreased signal resulted in a lower color filling rate.

The results of the present study also indicated that the color filling rates of images did not change significantly when the scan depth was altered. Changes in the depth altered the magnification of the images that appeared on the monitor, but did not alter the actual scanning parameters; thus there was no effect on the color filling rate.

A model phantom was used in the present study, which eliminated the effects of respiratory motion and individual differences that may be present in clinical studies. The model provided an effective tool for investigating the effects of different conditions on the image quality obtained with RULES. The position of the simulated gallbladder was superficial to allow its corresponding structure to be clearly shown using the linear array transducer. However, additional factors or multiple composite factors were not investigated in this study, which is a limitation that requires consideration when interpreting the obtained results. Furthermore, the preliminary results of this study require re-examination in vivo.

In conclusion, images obtained using RULES may be affected by instrument gain, and to a certain extent, by transducer frequency. Appropriate ranges of frequency and gain should be selected for RULES in clinical practice in order to obtain an optimal image and objective tissue characterization. The results of this study may be gallbladder specific, and require confirmation in vivo.

\section{Acknowledgements}

This study was supported by the International Cooperation Project of Shanxi Province (project no. 2008081040).

\section{References}

1. Halliwell M and Wells P (eds): Acoustical Imaging. Kluwer Academic/Plenum Publishers, New York, NY, 2000.

2. Ukimura $O$ and Gill IS (eds): Contemporary Interventional Ultrasonography in Urology. Springer-Verlag, London, 2009.

3. Donohue KD, Huang L, Burks T, Forsberg F and Piccoli CW: Tissue classification with generalized spectrum parameters. Ultrasound Med Biol 27: 1505-1514, 2001.

4. Masotti L, Biagi E, Breschi L, Granchi S, Di Lorenzo F and Magrini E: Tissue differentiation based on radiofrequency echographic signal local spectral content. Proc IEEE Ultrason Symp 1: 1030-1033, 2003.

5. Ponchietti R, Martorana G, Di Loro F, Bertaccini A, Nesi G, Grigioni WF, et al: A novel spectral ultrasonic differentiation method for marking regions of interest in biological tissue: in vitro results for prostate. Arch Ital Urol Androl 76: 147-153, 2004.

6. Scabia M, Biagi E and Masotti L: Hardware and software platform for real-time processing and visualization of echographic radiofrequency signals. IEEE Trans Ultrason Ferroelectr Freq Control 49: 1444-1452, 2002.

7. Schmitz G, Ermert H and Senge T: Tissue-characterization of the prostate using radio frequency ultrasonic signals. IEEE Trans Ultrason Ferroelectr Freq Control 46: 126-138, 1999.

8. Masotti L, Biagi E, Granchi S, Breschi L, Magrini E and Di Lorenzo F: Clinical test of Rules. IEEE Trans Ultrason Ferroelectr Freq Control 3: 2173-2176, 2004.

9. Bertaccini A, Franceschelli A, Schiavina R, Manferrari F, Brunocilla E, Marchiori D, et al: A novel spectral ultrasonic differentiation method for marking regions of interest in biological tissues. In vivo preliminary results. Arch Ital Urol Androl 79: 108-110, 2007.

10. Bertaccini A, Franceschelli A, Schiavina R, Marchiori D, Baccos A, Pernetti R, et al: Accuracy of a new echographic method (RULES, radiofrequency ultrasonic local estimators) in prostate cancer diagnosis. Anticancer Res 28: 1883-1886, 2008.

11. Masotti L, Biagi E, Granchi S, Luddi A, Breschi L and Facchini R: Carotid plaque tissue differentiation based on radiofrequency echographic signal local spectral content (RULES: Radiofrequency Ultrasonic Local Estimators). Biomedical Imaging: From Nano to Macro. ISBI 2008. 5th IEEE International Symposium on: 1051-1054, 2008. 